\title{
Représentation et manipulation des concepts mathématiques par le cerveau humain
}

\author{
Marie Amalric \\ Research group The Concepts, Actions, and Objects \\ (CAOs) Lab/The Kid Neuro Lab \\ Carnegie Mellon University Pittsburgh, USA
}

\begin{abstract}
Marie Amalric studies the localization of cognitive processes linked to mathematical activities. According to certain hypotheses, these processes are close to those concerned with language, while other hypotheses make them processes related to visuospatial processes. Marie Amalric first recalls previous work showing that we have from birth a minimal nucleus giving an intuitive approach to quantity, number, and positioning in space. These studies suggest that there is an innate proto-mathematical capacity, which then increases by learning. Then she presents experiments carried out comparatively with mathematician and non-mathematician subjects, submitting to them exercises in understanding statements of different types, mathematical and non-mathematical, and observing what is happening in the brain using fMRI imaging. The fMRI tests show that the areas involved in solving mathematical questions are different from those involved in the syntactic and semantic understanding of ordinary language. This is what is observed, as much with professional mathematicians as with non-mathematicians. They also show that at the level at which they can be observed, the same areas of the brain are called upon by mathematical questions, whatever their level and whatever the mathematical discipline concerned. Marie Amalric finally exposes additional investigations and their conclusions, concerning the localization of logical reasoning and the links with visuo-spatial processes. All of the experiments carried out would seem to indicate that the initial nucleus, which hosts proto-mathematical capacities from birth, then remains involved in the exercise of mathematics within a large sector which would grow around this initial nucleus.
\end{abstract}




\section{Des Intuitions « proto-mathématiques » fondamentales}

Des études comportementales ont révélé que les humains disposent, dès leur naissance, d'intuitions fondamentales relatives au nombre et à l'espace, intuitions qu'ils partagent avec de

\section{Théorie des "Core knowledge »: Cettethéorie développéepar ElizabethSpelke suggèreque} l'homme est muni de façon innée de 6 noyaux de connaissances fondamentales sur les

objets, les actions, les personnes, les nombres, les formes et l'espace navigable.

Ces systèmes sont encapsulés et initialement indépendants les uns des autres.

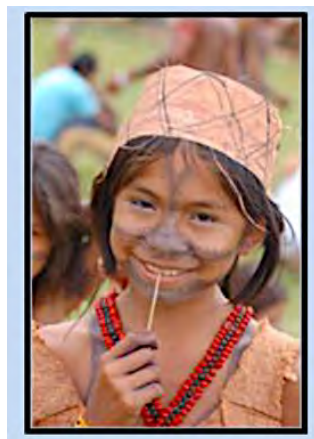

En Amazonie, les indiens Mundurukus disposent d'un vocabulaire mathématique pauvre et d'un accès limité à l'éducation.

nombreuses autres espèces animales. En particulier, des bébés âgés de quelques heures sont déjà capables d'extraire des informations numériques de leur environnement. Izard et collaborateurs (2009) ont ainsi montré qu'après avoir été exposés à des séries de sons contenant un certain nombre de syllabes, ces nouveau-nés manifestaient plus d'intérêt pour une image montrant le même nombre d'objets que pour une image montrant un nombre d'objets différent. Très rapidement au cours du développement, les bébés se révèlent dotés de la capacité de percevoir de manière exacte des quantités inférieures à 3 (c'est la subitisation), ainsi que de comparer et ajouter des quantités de manière approximative (Wynn, 1992). La présence de capacités similaires a pu être identifiée chez de très nombreuses espèces animales, à commencer par les singes et plus généralement les mammifères tels que les félins ou les chevaux, mais aussi chez les poissons, les oiseaux, les amphibiens et même les insectes (Benson-Amram et al., 2011; Rugani et al., 2009). De façon remarquable, la perception numérique chez les différentes espèces animales partage une caractéristique commune : un effet de distance mesuré sur une échelle logarithmique, qui traduit le fait que plus des nombres sont grands et proches, plus il est difficile de les comparer (Cantlon et al., 2016). Très récemment, il a été montré que cet effet de distance s'étend également au traitement non-symbolique des ratios (Matthews et al., 2016). La perception spontanée que les humains semblent avoir des ratios pourrait d'ailleurs sous- tendre une autre compétence mathématique qui émerge très précocement chez l'homme : une sensibilité certaine aux probabilités et aux régularités statistiques (Marcus et al., 1999; Teglas et al., 2011). Enfin, l'homme s'avère être muni, comme beaucoup d'autres espèces animales, d'intuitions spontanées des relations spatiales, mais aussi des formes et de leurs propriétés. Dans des tâches de réorientation spatiale où l'objectif est de localiser un objet, les très jeunes enfants et de nombreuses espèces animales se repèrent et s'orientent principalement grâce à des indices géométriques de distance et de direction (Chiandetti and Vallortigara, 2007; Lee and Spelke, 2008). De plus, malgré l'absence d'éducation formelle, les jeunes enfants américains et les adultes Mundurukus se 
montrent spontanément capables d'extraire et d'utiliser les informations géométriques abstraites contenues dans une carte pour localiser un objet, même lorsqu'ils y sont confrontés pour la première fois (Dillon et al., 2013). Par ailleurs, des tâches de détection d'intrus ont été utilisées pour montrer que l'absence d'éducation ou de vocabulaire numérique n'empêche en rien les Mundurukus de reconnaitre spontanément de nombreux concepts géométriques tels que des formes et des propriétés euclidiennes, topologiques ou métriques (Dehaene et al., 2006). Ces intuitions forment des systèmes de connaissances fondamentales, ou « core knowledge » selon la théorie du même nom développée par Elizabeth Spelke.

\section{La « bosse des maths » revisitée}

Le récent développement des techniques d'imagerie cérébrale a offert un éclairage nouveau sur les bases cérébrales de ces intuitions fondamentales. L'utilisation de la technique d'imagerie par résonance magnétique fonctionnelle (IRMf) a notamment mené à l'hypothèse que le sillon intrapariétal puisse jouer un rôle décisif dans la représentation des nombres. Il semble en effet systématiquement impliqué dans la réalisation de tous types de tâches numériques (calcul, comparaison, approximation, avec des symboles ou non,...) chez les adultes mais aussi chez de très jeunes enfants (Dehaene et al., 2003; Hyde et al., 2010; Piazza et al., 2004). Une étude très récente a même suggéré l'existence d'une certaine « numérotopie » dans le lobule pariétal supérieur (Harvey et al., 2013). Cela signifie

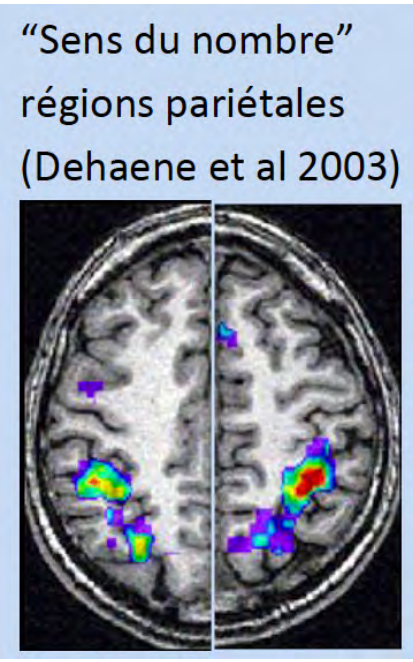
que les réponses corticales à des

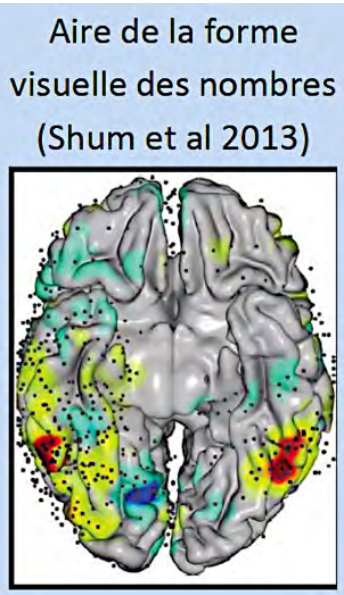
nombres voisins se situent aussi dans des régions voisines, rangées dans le même ordre que les nombres auxquels ces régions répondent. De plus, des études intracrâniennes ont révélé que des régions pariétales équivalentes semblent également être impliquées dans la réalisation de tâches numériques chez le singe (Nieder and Miller, 2004). Chez l'homme, les études intracrâniennes sont bien entendu rares, et cantonnées aux cas de patients, le plus souvent épileptiques. Mais notamment deux d'entre elles ont permis de révéler que des électrodes localisées dans le gyrus temporal inférieur répondaient davantage à la présentation de chiffres arabes que de lettres ou de faux symboles (Shum et al., 2013), et davantage au calcul qu'à la lecture de phrases (Hermes et al., 2015). Les régions cérébrales ainsi identifiées ont été nommées « aires de la forme visuelle des nombres ». 
Cependant, les mathématiques ne peuvent se résumer à la compréhension basique des nombres et de l'espace, et les mécanismes par lesquels le cerveau humain parvient à conceptualiser des objets mathématiques plus avancés restent obscurs. Notamment, le rôle du langage dans le développement des connaissances mathématiques est très débattu en sciences cognitives

\section{La pensée mathématique peut-elle exister sans langage ?}

Voici une question séculaire qui intrigue bien des philosophes et scientifiques. L'activité mathématique et les compétences pour le langage parlé reposent pour certains sur les mêmes mécanismes d'abstraction. Noam Chomsky (2006) prétend même que l'activité mathématique a émergé chez l'Homme comme conséquence de ses capacités de langage. Pourtant, la plupart des mathématiciens et physiciens ne perçoivent pas l'influence du langage dans leur réflexion. C'est ce qu'évoque notamment le mathématicien français Jacques Hadamard en 1945, dans un ouvrage analysant les processus à l'œuvre dans l'invention mathématique. En particulier, il y rapporte sa correspondance avec nombre de ses collègues, dont Albert Einstein, qui lui écrit : «Les mots ou le langage, écrit ou parlé, ne semblent pas jouer le moindre rôle dans le mécanisme de ma pensée. Les entités psychiques qui servent d'éléments à la pensée sont certains signes ou des images plus ou moins claires [...] qui sont, dans mon cas, de type visuel et parfois moteur. Les mots [...] n'ont à être cherchés avec peine qu'à un stade secondaire. »

En accord avec l'idée de Noam Chomsky, quelques études tendent à montrer que les mots de nombres sous-tendent le développement des compétences numériques exactes. Par exemple, les Mundurukus sont capables de réaliser des additions approximatives aussi bien que les Français, mais ils échouent lorsqu'il s'agit de réaliser des soustractions exactes dont le résultat ne dépasse pourtant pas le seuil de subitisation (Pica et al., 2004).

D'un autre côté, plusieurs études ont suggéré que l'arithmétique et le calcul algébrique simple sont dissociés du langage parlé naturel. C'est le cas chez des patients aphasiques, qui présentent des troubles de la compréhension ou de la production du langage, mais qui peuvent toujours faire des calculs ou de l'algèbre (Varley et al., 2005). A l'inverse, une acalculie acquise, suite à un AVC par exemple, peut laisser les capacités de langage intactes (Dehaene and Cohen, 1995). Quelques études d'IRM ont également révélé des différences nettes d'activations cérébrales pour les activités algébriques versus linguistiques (Monti et al., 2012). Une alternative à l'hypothèse de Chomsky est donc que l'arithmétique se construit directement sur les intuitions proto-mathématiques du nombre, et qu'au niveau cérébral, elle recycle certaines régions associées au traitement numérique de base. Certaines études plaident en faveur de cette hypothèse, en montrant qu'au niveau individuel, l'acuité du "sens du nombre" prédit les compétences mathématiques ultérieures (Halberda et al., 2008). 
Alors que les études passées se sont principalement intéressées à l'arithmétique élémentaire, mon travail de thèse a, pour la première fois, permis de mettre en place une série d'expériences visant à déterminer quelles aires cérébrales sont impliquées dans la réflexion mathématique de haut niveau.

\section{Origine cérébrale des concepts mathématiques de haut niveau (Amalric and Dehaene, 2016)}

Dans une première étude en IRMf à haute résolution, 15 mathématiciens professionnels (chercheurs ou professeurs), et 15 sujets contrôles ayant le même niveau universitaire mais n'ayant pas fait d'études mathématiques au-delà du lycée (chercheurs ou professeurs en littérature, histoire, linguistique, etc...), ont écouté 72 affirmations mathématiques (en analyse, algèbre, topologie et géométrie) et non-mathématiques de haut niveau et ont dû déterminer en quelques secondes si elles étaient vraies, fausses ou dépourvues de sens.

Son

d'alerte

(b)

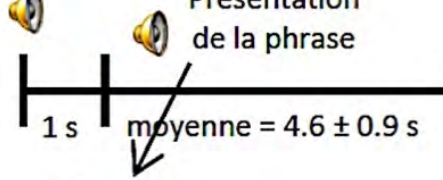

Exemples:

Math: "Une mesure finie invariante à gauche sur un groupe compact est bi-invariante."

Non-Math: "En Grèce antique, un citoyen incapable de payer ses dettes devenait esclave."

Figure 1 Schéma d'essai d'un protocole auditif de l'expérience 1

Les résultats comportementaux ont permis de vérifier que les affirmations mathématiques et non-mathématiques avaient des difficultés équivalentes. En effet, le taux de bonnes réponses des mathématiciens aux affirmations mathématiques et non-mathématiques étaient identiques. Dans un second temps, des images de maisons, outils, visages, corps humains, mots, nombres, fragments d'équations et damiers circulaires (servant de contrôle pour l'activation des aires visuelles primaires) ont été présentées par blocs de 8 images d'une même catégorie. Les participants ont dû appuyer sur un bouton lorsqu'ils détectaient la répétition consécutive d'une même image.

\begin{tabular}{|llllll}
\hline Bodies Tools Faces Houses Words Numbers Equations & Checkers \\
\hline
\end{tabular}

Figure 2 Catégories visuelles 
Enfin, dans les 5 dernières minutes de l'examen IRM, les participants ont, entre autres, fait du calcul mental simple (des soustractions présentées oralement ou visuellement) et lu ou écouté des phrases non-mathématiques simples (voir (Pinel et al., 2007) pour plus de détails).

Le contraste des affirmations mathématiques moins les affirmations non-mathématiques pendant la phase de réflexion a alors permis de mettre au jour un ensemble d'aires cérébrales (en rouge sur la figure 3 ) impliquées dans la réflexion mathématique de haut niveau chez les mathématiciens professionnels. Cet ensemble est constitué, bilatéralement, des sillons intrapariétaux, de régions temporales inférieures et de régions frontales. L'analyse du décours temporel de l'activation au sein de chacune de ces régions a révélé qu'elles étaient systématiquement activées par chacun des domaines mathématiques testés, analyse, algèbre, topologie et géométrie. Au contraire, aucune activation n'y était visible en réponse aux affirmations non-mathématiques.

De plus, cet ensemble de régions cérébrales n'a été identifié dans cette expérience que chez les mathématiciens. En effet, les affirmations mathématiques ont été traitées de manière similaire aux affirmations non-mathématiques dépourvues de sens par les sujets contrôles. Cela n'est pas surprenant, mais tend à montrer qu'il ne suffit pas de savoir que le contenu présenté est un contenu mathématique pour entraîner une activation des régions cérébrales ci-dessus.

En revanche, aucune différence n'a été observée entre nos deux groupes de participants lorsqu'il s'agissait de faire du calcul mental simple. En accord avec de précédentes études, nous avons confirmé que cette tâche entraîne notamment une activation des sillons intrapariétaux bilatéraux et de régions préfrontales.

De plus, nous avons pu observer, pour la première fois en IRMf, une activation des régions temporales inférieures désignées comme " aires de la forme visuelle des nombres », en réponse à cette tâche de calcul mental (cf. la carte cérébrale bleue de la figure 3). Par ailleurs, la simple exposition visuelle à des images de nombres versus toutes les autres catégories visuelles présentées entrâne également des activations cérébrales similaires chez les deux groupes de participants.

Ces activations se situent, à nouveau, dans les sillons intrapariétaux bilatéraux, des régions préfrontales et des régions temporales inférieures bilatérales (cf. la carte verte de la figure 3). Comme le révèle la figure 3 , toutes les tâches portant sur un contenu mathématique dans cette expérience activent des régions dont le recouvrement et l'intersection sont remarquables. En d'autres termes, la réflexion mathématique sur des concepts de haut niveau semble activer les mêmes régions cérébrales que des tâches mathématiques beaucoup plus basiques. Nous avons par ailleurs vérifié que ce recouvrement n'était pas simplement dû à la présence de nombres au sein des affirmations mathématiques. Bien que nous ayons été très vigilants à ce que nos phrases ne contiennent aucune mention directe de nombres, certaines présentaient une référence indirecte aux nombres ou aux fractions 
(ex: $\mathbb{R} 2$, sphère unité, demi grand axe, etc). Après avoir éliminé toutes ces affirmations, nous avons donc ré-analysé le contraste math > non- math et avons obtenu des résultats tout à fait comparables à ceux précédemment obtenus. De plus, nous avons réalisé une analyse dite « representational similarity » (RSA) à l'échelle individuelle. Au sein d'une région donnée, identifiée comme répondant aux mathématiques à l'échelle du groupe, nous avons vérifié que l'activation entraînée par la réflexion mathématique était plus semblable à l'activation entraînée par les autres stimuli mathématiques (nombres, formules et calcul mental) qu'à l'activation entrânée par les stimuli (phrases et images) non-mathématiques.

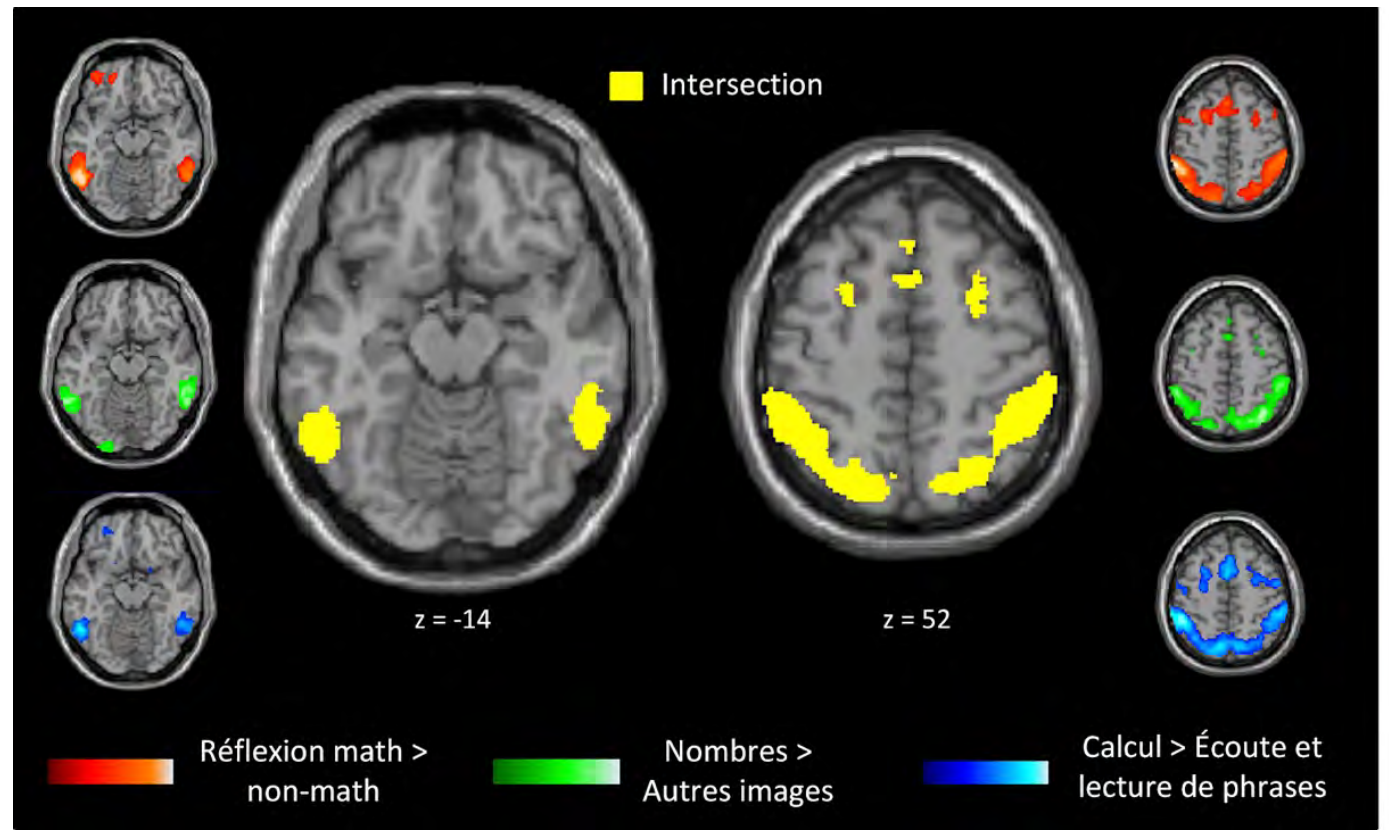

Figure 3 Cartes montrant les contrastes principaux de l'expérience 1 et leur intersection

Ces observations cö̈ncident avec la théorie du recyclage neuronal, développée par Stanislas Dehaene, et qui stipule que les activités culturelles de haut niveau, telles que les mathématiques, recyclent des fondations cérébrales très anciennes dans l'évolution, telles que le sens du nombre, de l'espace ou du temps.

\section{Organisation de la voie visuelle ventrale chez les mathématiciens professionnels (Amalric and Dehaene, 2016)}

L'analyse des contrastes de chaque catégorie d'images versus toutes les autres a révélé une mosaïque typique de réponses préférentielles à chaque catégorie dans le cortex occipitotemporal ventral chez nos deux groupes de participants. Nous avons en particulier observé 
la zone de reconnaissance des visages du gyrus fusiforme droit (FFA, en rouge sur la figure 4), les zones parahippocampiques bilatérales de reconnaissance des lieux (PPA, en jaune), les zones de reconnaissance des corps humains du cortex extrastrié (EBA, en orange), les cortex occipitaux latéraux bilatéraux de reconnaissance des outils (LOC, en violet) et l'aire de la forme visuelle des mots dans l'hémisphérique gauche (VWFA, en rose). Comme cela a déjà été évoqué plus haut, nous avons également trouvé, grâce à l'IRMf à haute résolution, une forte activation liée au nombre dans les régions bilatérales du gyrus temporal inférieur (en bleu), aux sites correspondants aux zones de la forme visuelle des nombres gauche et droite (VNFA). Nous avons également observé des réponses préférentielles aux formules (en vert) chez nos deux groupes, à des sites bilatéraux chevauchant partiellement la VNFA.

Étant donné que le traitement des mathématiques avancées recrute des aires ventrales du gyrus temporal inférieur, une question était de savoir dans quelle mesure l'activation de ces régions varie avec l'expertise mathématique. Chez les mathématiciens, par rapport aux sujets contrôles, nous avons observé : (1) une augmentation de l'intensité et de l'étendue de la réponse aux formules mathématiques dans le gyrus temporal inférieur gauche ; (2) dans une moindre mesure une augmentation de l'intensité de la réponse aux nombres au pic de la VNFA gauche; (3) une diminution de la réponse aux visages dans l'hémisphère droit.

Ces résultats soutiennent à nouveau l'idée d'une forme de recyclage neuronal de régions qui préexistent. Les représentations des nombres s'inscrivent ainsi, chez tous, dans des sous-régions occipito-temporales ventrales, remarquablement dissociées de l'expertise pour la lecture. Puis l'expertise mathématique entraine l'acquisition de symboles nouveaux dont la représentation vient augmenter l'activité de ces sous-régions.

\section{Mathématiques et langage sont sémantiquement distincts dans le cerveau (Amalric and Dehaene,2018, 2016, submitted)}

Cette première expérience a également montré que, bien que les affirmations mathématiques présentées prennent la forme de phrases, les aires cérébrales activées par la réflexion sur des problèmes mathématiques (en bleu sur la figure 4) ne présentaient aucun recouvrement avec les aires du langage (identifiées indépendamment par l'activation engendrée par la lecture et l'écoute de phrases non-mathématiques simples dans la dernière partie de l'expérience 1). A l'inverse, lorsque la réflexion des mathématiciens portaient sur des problèmes d'histoire ou de géographie, le réseau d'aires cérébrales qui s'activaient (en vert sur la figure 4) était complètement différent des régions mathématiques et impliquait certaines aires du langage. Cette observation a ensuite été confirmée par la réalisation d'une analyse fine, à l'échelle individuelle, de l'activation entraînée par les affirmations mathématiques et non-mathématiques au sein de 7 régions d'intérêt présentant une réponse à la syntaxe de la langue d'après l'étude de Pallier et collaborateurs (2011). Dans chacune de ces régions, nous avons pu vérifier que les affirmations non-mathématiques entraînaient plus d'activation que les affirmations mathématiques. 


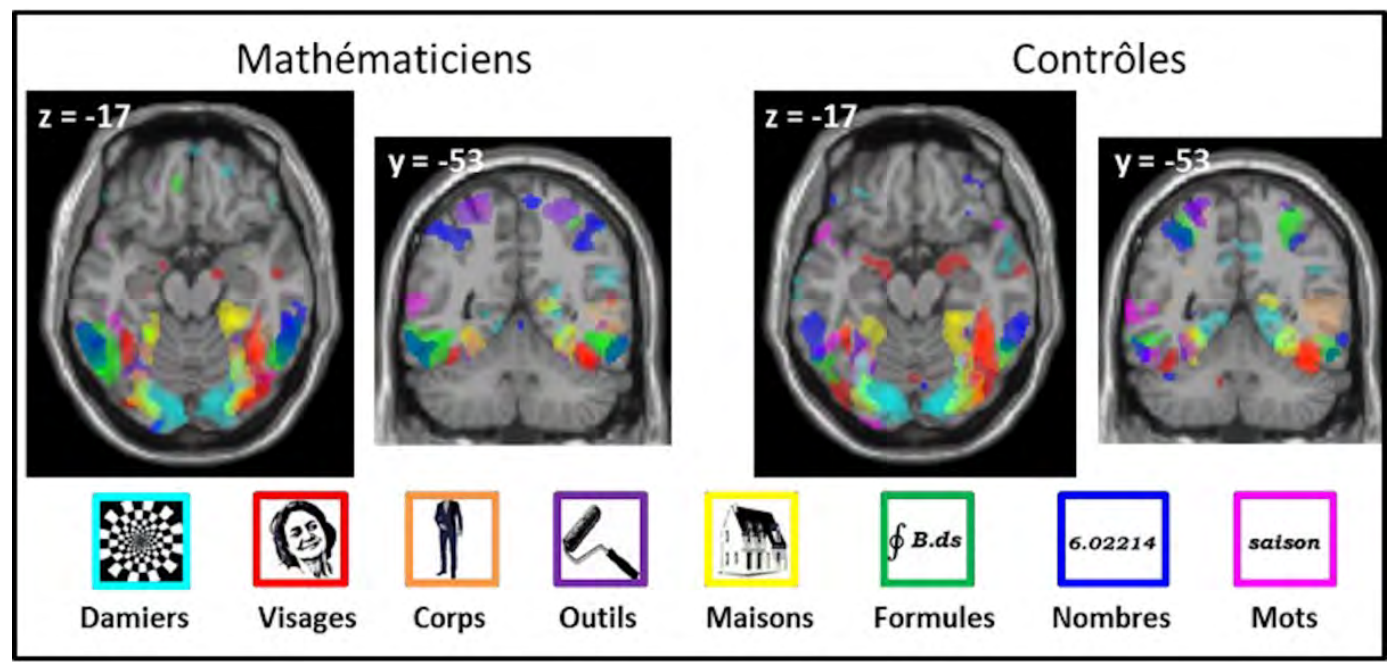

Figure 4 Mosaïque ventrale de réponses préférentielles aux catégories d'images présentées dans l'expérience 1

Nous avons donc observé une séparation complète entre le traitement cérébral des affirmations mathématiques et le traitement cérébral des affirmations non-mathématiques, et nous avons dans un deuxième temps voulu tester si cette séparation pouvait émaner du seul contenu sémantique des affirmations. Dans deux nouvelles expériences, nous avons testé plusieurs alternatives possibles à cette hypothèse. D'abord, après avoir constaté que le réseau d'aires qui répond aux mathématiques coïncide en partie avec le "système de demande multiple » décrit par Duncan (2010), une question légitime était de savoir si les problèmes mathématiques requièrent intrinsèquement plus d'attention et de ressources cognitives que les problèmes non-mathématiques. A l'inverse, se pourrait-il que certains problèmes mathématiques connus par cour ou reposant sur la seule visualisation de la solution court-circuitent le réseau d'aires répondant aux mathématiques? Par ailleurs, les différences syntaxiques entre les problèmes mathématiques et non-mathématiques de notre première expérience pourraient-elles induire des stratégies de résolution intrinsèquement différentes? Enfin, quels facteurs déterminent ce qui active les réseaux du langage et des mathématiques? L'algèbre est-elle intrinsèquement reliée au traitement du langage? La présence d'opérateurs logiques ou numériques suffit-elle à activer le réseau répondant aux mathématiques même dans des phrases non-mathématiques

Un nouveau groupe de 14 mathématiciens professionnels a donc participé à deux expériences contrôles en IRMf, basées sur un protocole tout à fait similaire à l'expérience 1 : les participants devaient écouter des affirmations mathématiques et non-mathématiques et décider si elles étaient vraies ou fausses. Cette fois, ils ne disposaient que de 2.5 secondes à l'issue de la présentation de la phrase pour répondre, et plus aucune affirmation n'était dépourvue de sens. De plus, les affirmations ne consistaient qu'en des faits mathématiques 
et non-mathématiques simples. Dans l'expérience 2, nous avons proposé aux participants de réfléchir à des faits algébriques connus par cœur (telles que les identités remarquables), du calcul algébrique simple, des problèmes de trigonométrie exprimés avec des cosinus et sinus ou avec des nombres complexes et nécessitant la visualisation du cercle trigonométrique, des problèmes de géométrie non métrique, et des faits non-mathématiques simples portant sur les arts. Dans l'expérience 3, nous avons réduit les affirmations à leur plus simple expression en utilisant seulement l'auxiliaire être, et avons été très vigilants à ce que la syntaxe des affirmations mathématiques et non-mathématiques soit strictement identique. Nous avons inclus de simples déclaratives affirmatives (« La fonction sinus est périodique »; «Les bus londoniens sont rouges »), quantifiées (« Certaines matrices sont diagonalisables »; «Certains romans sont autobiographiques »), négatives (« La fonction exponentielle n'est pas constante »; « La forêt amazonienne n'est pas désertique »), négatives et quantifiées («Certains ensembles finis ne sont pas dénombrables » « Certaines plantes vertes ne sont pas grimpantes »).

La figure 5 montre que dans ces deux expériences, la dissociation de traitement cérébral des affirmations mathématiques et non-mathématiques est répliquée, même lorsque cellesci sont très simples, et même lorsque leur syntaxe est parfaitement identique. Autre fait remarquable, les activations préfrontales observées dans l'expérience 1 pour le contraste math > non-math semblent disparaître à mesure que les affirmations deviennent plus simples. Les sillons intrapariétaux et les régions inférieures temporales semblent, eux, constituer un noyau d'aires systématiquement activées par les mathématiques. Un nouveau type d'analyse, consistant à moyenner sur plusieurs régions d'intérêt l'intensité d'activation pour chacune des affirmations, a révélé qu'aucune affirmation nonmathématique n'entraîne l'activation du sus-désigné noyau d'aires cérébrales. À l'inverse, dans les régions d'intérêt liées à la syntaxe du langage, certains domaines mathématiques tels que l'algèbre ou la trigonométrie n'entraînent que très peu voire pas du tout d'activation.

Par ailleurs, nous avons pu vérifier que même les problèmes mathématiques connus par cœur ou nécessitant une certaine imagerie visuelle ne court-circuitent pas pour autant le réseau d'aires cérébrales activé par les mathématiques. Au contraire, toutes les catégories testées dans l'expérience 2 entraînent des activations similaires dans les sillons intrapariétaux et les régions temporales inférieures.

Enfin, les opérateurs logiques minimaux tels que les quantifieurs et la négation n'entraînent pas à eux seuls l'activation des régions répondant aux mathématiques : l'effet principal des quantificateurs se situe dans le gyrus angulaire droit, et l'effet principal de la négation se situe dans une région frontale inférieure gauche, deux régions qui sont en dehors du réseau répondant aux mathématiques.

Tous ensembles, ces résultats soutiennent l'idée que la sémantique mathématique est fondamentalement dissociée du reste de la sémantique dans le cerveau, et repose sur un 
noyau d'aires cérébrales constitué des sillons intrapariétaux et de régions temporales inférieures.

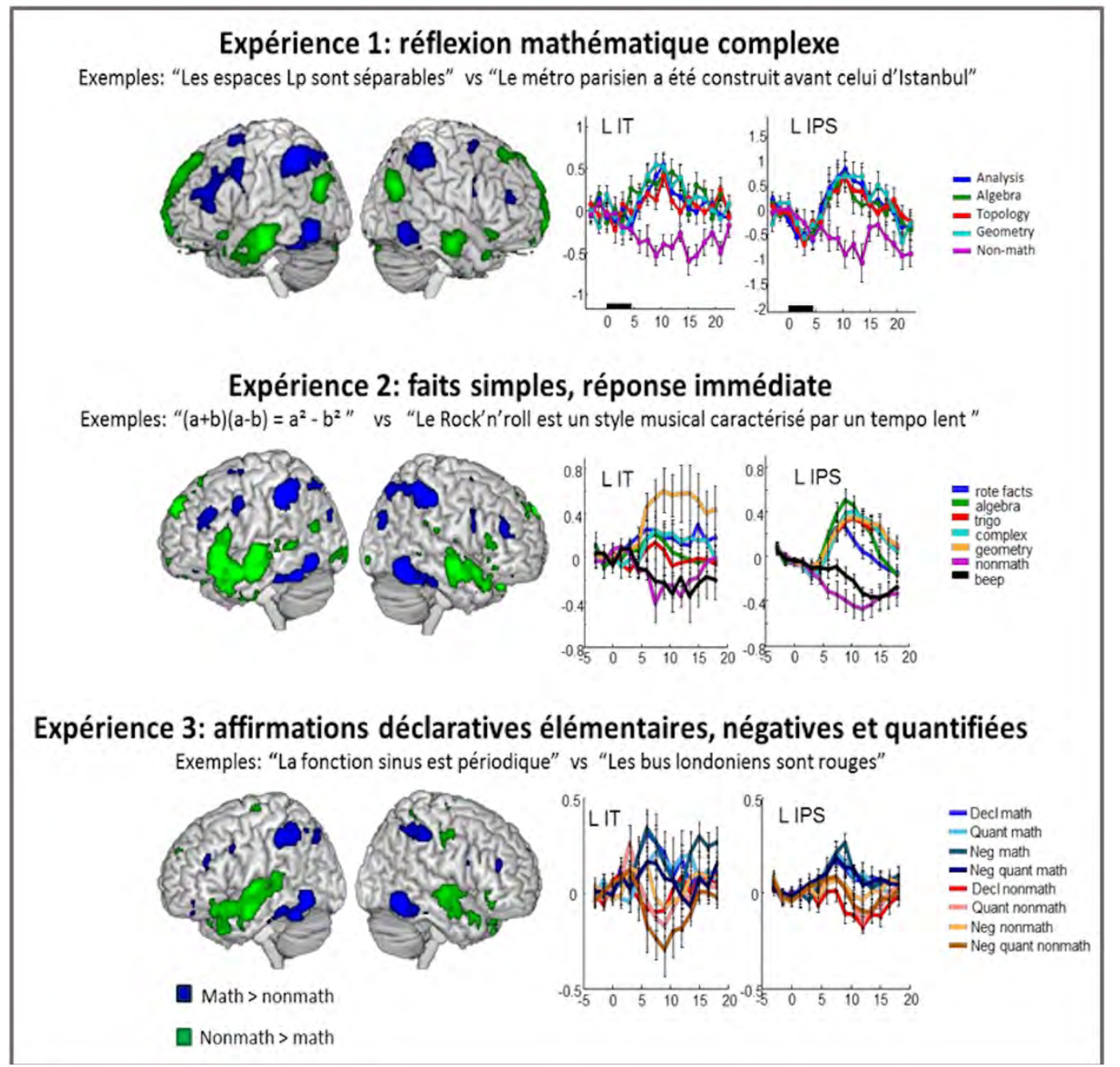

Figure 5 Comparaison des contraste math-> non-math et non-math>math dans les trois expériences d'IRMf et décours temporels de l'activation dans les principales régions répondant aux mathématiques

\section{Un langage mathématique sans mots pour le dire (Amalric et al., 2017b ; Wang et al, submitted)}

Ce résultat concorde avec d'autres observations. Notamment, disposer d'un vocabulaire mathématique ne se révèle pas être absolument nécessaire à la compréhension de notions 
mathématiques. Typiquement, les études menées en Amazonie auprès des indiens Mundurukus, qui disposent d'un vocabulaire numérique et géométrique très pauvre et d'un accès limité à l'éducation, ont révélé qu'ils sont tout à fait capables de réaliser des opérations arithmétiques, ou d'identifier des propriétés géométriques du plan et de l'espace (Dehaene et al., 2008, 2006; Pica et al., 2004). Ces études suggèrent l'existence de primitives des mathématiques en l'absence de langage. On peut ne pas savoir nommer un carré et pourtant posséder le concept de carré.

La question qui se pose alors est de savoir s'il existe également un langage mathématique élémentaire, même lorsque l'on n'a pas été éduqué, même lorsque l'on n'a pas de mots pour le dire. Au cours de ma thèse, j'ai conçu une situation suffisamment simple pour être présentée à de jeunes enfants ou à des indiens Mundurukus, mais qui requiert une sorte de langage de l'esprit (Fodor, 1975), en l'occurrence un « langage de la géométrie ». Cette situation demande de regarder une séquence spatiale présentant un certain nombre de positions successives, d'en prédire la suite et d'en mémoriser l'ensemble. La prédiction s'appuie sur la détection de régularités géométriques dans la séquence : symétries, rotations, formes... (cf. figure 6) Et la présence de régularités facilite également la mémorisation.

La tâche proposée aux sujets consistaient, après avoir vu les premiers points de la séquence, à désigner les suivants. À chaque erreur, la séquence recommençait au début jusqu'à corriger le point erroné. Afin de tester l'existence d'un langage géométrique spontané, il était important que les participants soient non seulement des adultes français $(n=23)$, mais aussi des enfants de 5 ans qui n'ont pas encore eu d'éducation mathématique (n=47), ainsi que des adultes Mundurukus d'Amazonie qui n'ont pas eu d'éducation mathématique du tout $(\mathrm{n}=14)$.

En se servant de la séquence « irregular », qui ne présente aucune régularité apparente, comme référence du degré d'apprentissage par cœur des séquences, l'analyse des erreurs commises à des points clés des séquences « repeat », « alternate », « repeat +2 », « 4 segments » et « 4diagonals » a permis de montrer que tous les participants sont capables d'identifier et d'utiliser rapidement des rotations et des symétries axiales afin de compléter correctement les séquences avant même qu'elles n'aient été entièrement présentées. Seule la symétrie centrale a semblé plus difficile à comprendre en l'absence d'éducation (chez les enfants et les Mundurukus). De plus, l'analyse des erreurs commises aux points 5 et 13 des séquences «2arcs » et «2squares » (qui correspondent à l'application de la règle indiquant comment changer le point de départ de l'arc ou du carré), ont montré que tous les participants sont également capables de détecter rapidement des structures enchâssées. Les séquences « 2rectangles » et « 2crosses » qui contiennent un niveau d'enchâssement supplémentaire se sont révélées plus difficiles pour les adultes français et Mundurukus, voire complètement inintelligibles pour les jeunes enfants. Nous avons ainsi pu montrer que le langage requis pour décrire les séquences observées, contient à la fois des primitives géométriques (symétries, rotations) et la capacité de combiner ces primitives sous forme de règles de répétition. 


(2)eat+1

Figure 6 Séquences présentées aux adultes (en vert et en bleu) et aux enfants (en vert et en jaune)

Dans ce langage formel, chaque séquence a une certaine complexité, associée à la longueur de sa description minimale (Romano et al., 2013). Nous avons pu vérifier que notre langage géométrique et les complexités des séquences prédisent efficacement les erreurs commises par les participants dans les tâches de prédiction et de mémorisation des séquences (cf. figure 7).

À partir du langage géométrique ainsi créé, nous avons ensuite développé un modèle computationnel de l'apprentissage des séquences géométriques. Comme représenté sur la figure 8, l'algorithme prend en entrée les premiers points de la séquence présentés au sujet, puis crée la liste de toutes les séquences possibles commençant par ces points et leur associe leur expression (appelée programme sur la figure 8) dans le langage prédéterminé. Pour refléter le fait que plus la longueur de l'expression augmente, plus la probabilité que les sujets se trompent est grande, notre algorithme évalue la complexité de chaque programme avec un bruit gaussien. L'algorithme choisit ensuite le programme qui minimise la complexité. Afin d'éviter que la performance au dernier point soit toujours parfaite, on suppose que le modèle ne peut identifier des expressions qu'en deçà d'une complexité seuil. Au-delà, le point suivant est choisi aléatoirement. Sinon, le point suivant est défini par le programme choisi. 

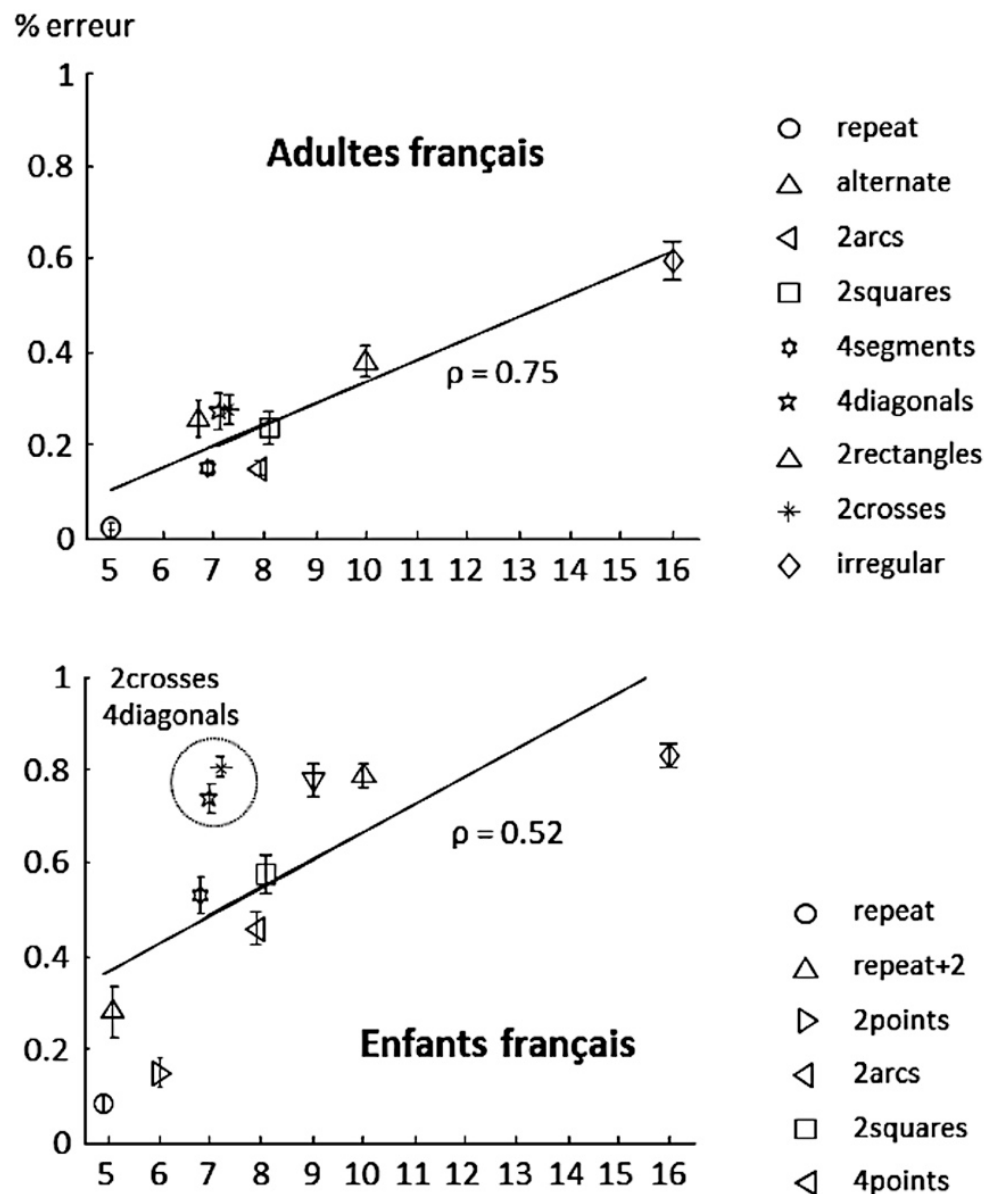

- repeat

$\triangle$ repeat+2

$\triangleright$ 2points

$\triangleleft 2$ 2arcs

$\square \quad$ 2squares

$\triangleleft$ 4points

\& 4segments

๖ 4diagonals

$\triangle 2$ rectangles

* 2crosses

$\diamond \quad$ irregular

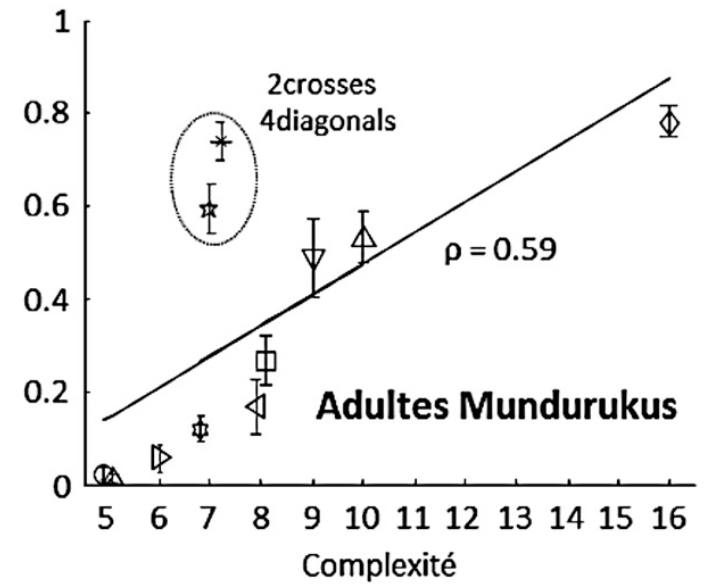

Figure 7 Corrélation du taux d'erreur avec la complexité des séquences 


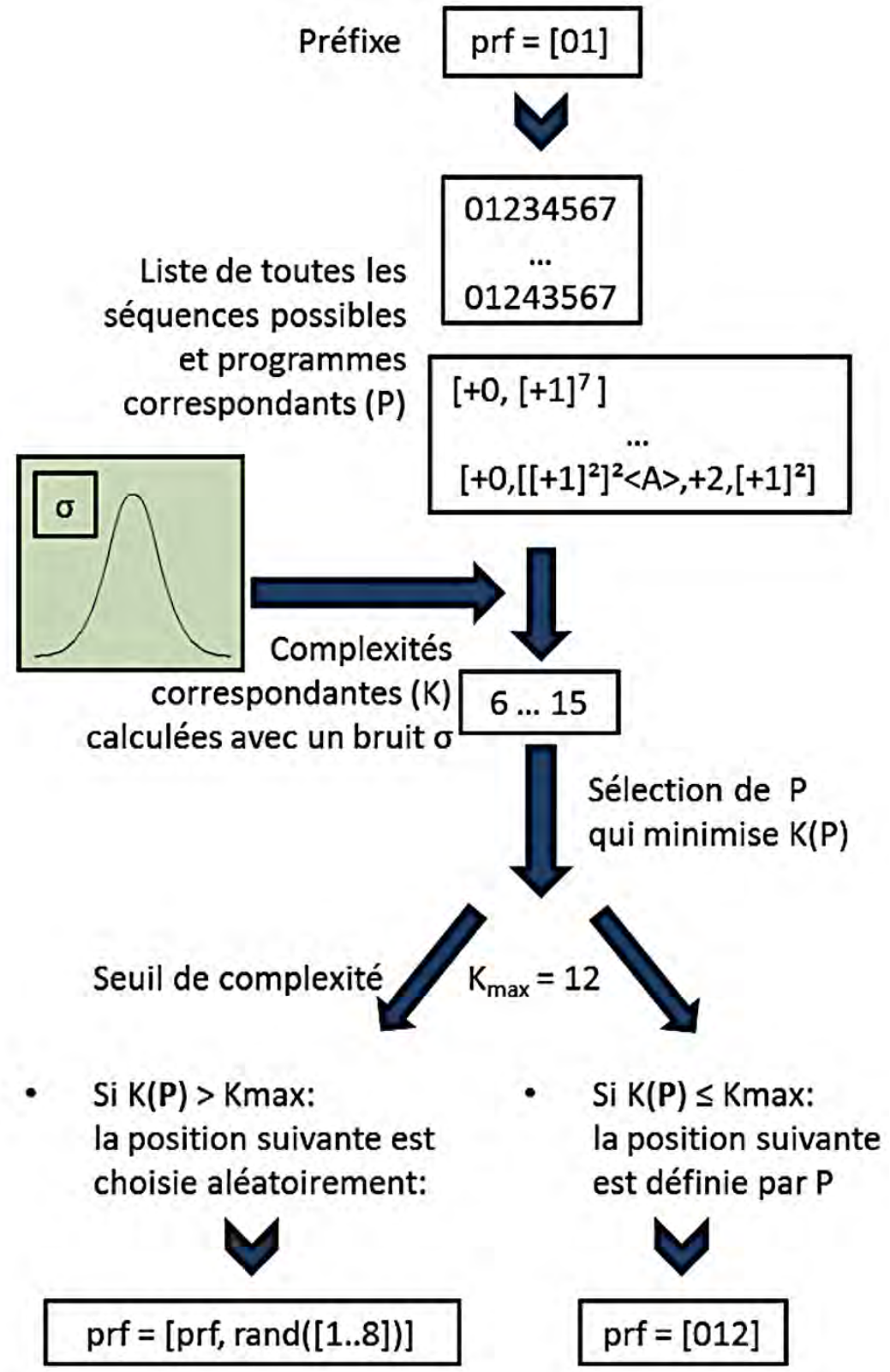

Figure 8 Schéma de l'algorithme utilisé 
Pour évaluer ce modèle, nous avons seulement considéré les 8 séquences présentées dans tous les groupes (en vert sur la figure 6) et qui ne présentent pas de répétition interne. La détermination du seul paramètre $\sigma$ a suffi à modéliser les données des adultes de manière tout à fait remarquable. En revanche, pour reproduire le comportement des enfants et des Mundurukus de façon satisfaisante, il a fallu considérer un langage restreint, sans symétrie centrale et sans la capacité d'encoder plus d'un niveau de répétition.

Ces résultats semblent indiquer d'une part que le cerveau humain possède une forme de langage de la pensée géométrique indépendant de tout langage parlé naturel, présent spontanément mais qui se raffine avec l'âge et l'éducation, et d'autre part que le cerveau cherche dans ce langage l'expression la plus courte possible qui permet de rendre compte de ce qui a été observé.

Afin de tester quelles régions cérébrales s'activent au cours de l'apprentissage des séquences, Liping Wang et moi avons ensuite adapté ce protocole comportemental à l'expérimentation en IRMf. Nous avons proposé à 20 adultes français non plus de désigner les positions successives, mais de suivre un point mobile du regard en essayant d'anticiper sa position. Grâce à un dispositif de suivi oculaire, nous avons pu calculer un indice d'anticipation du regard, et avons vérifié que les effets d'anticipation étaient un bon marqueur de la compréhension implicite des séquences. En effet, les données oculaires étaient tout à fait similaires aux données issues du pointage, et l'indice d'anticipation global était fortement corrélé à la complexité des séquences.

Nous avons par la suite étudié dans quelles régions du cerveau l'activité corrélait avec la complexité des séquences. Après avoir éliminé l'effet de la mémoire de travail, nous avons pu observer une activation dans la partie supérieure du gyrus frontal inférieur, région qui appartient au réseau impliqué dans le traitement mathématique identifié dans mes précédentes expériences.

Nous avons ensuite quantifié l'anticipation des structures enchâssées, en faisant la différence entre les points 5 , qui correspondent à la règle de second niveau, et les points 3 et 7 , qui correspondent à la règle de premier niveau. Les régions cérébrales dont l'activité corrélait avec cet indice sont représentées en jaune sur la figure 9 et incluent des régions pariétales bilatérales, préfrontales et temporales inférieures droites. En comparant l'intensité de cette activation à l'intérieur de régions d'intérêt du langage (en rouge) et des mathématiques (en bleu), nous avons finalement observé que l'anticipation de l'enchâssement repose davantage sur l'activation du réseau de traitement mathématique que du réseau de traitement linguistique.

Ces résultats suggèrent l'existence d'un "langage de la pensée » de nature géométrique, indépendant du langage parlé naturel. 


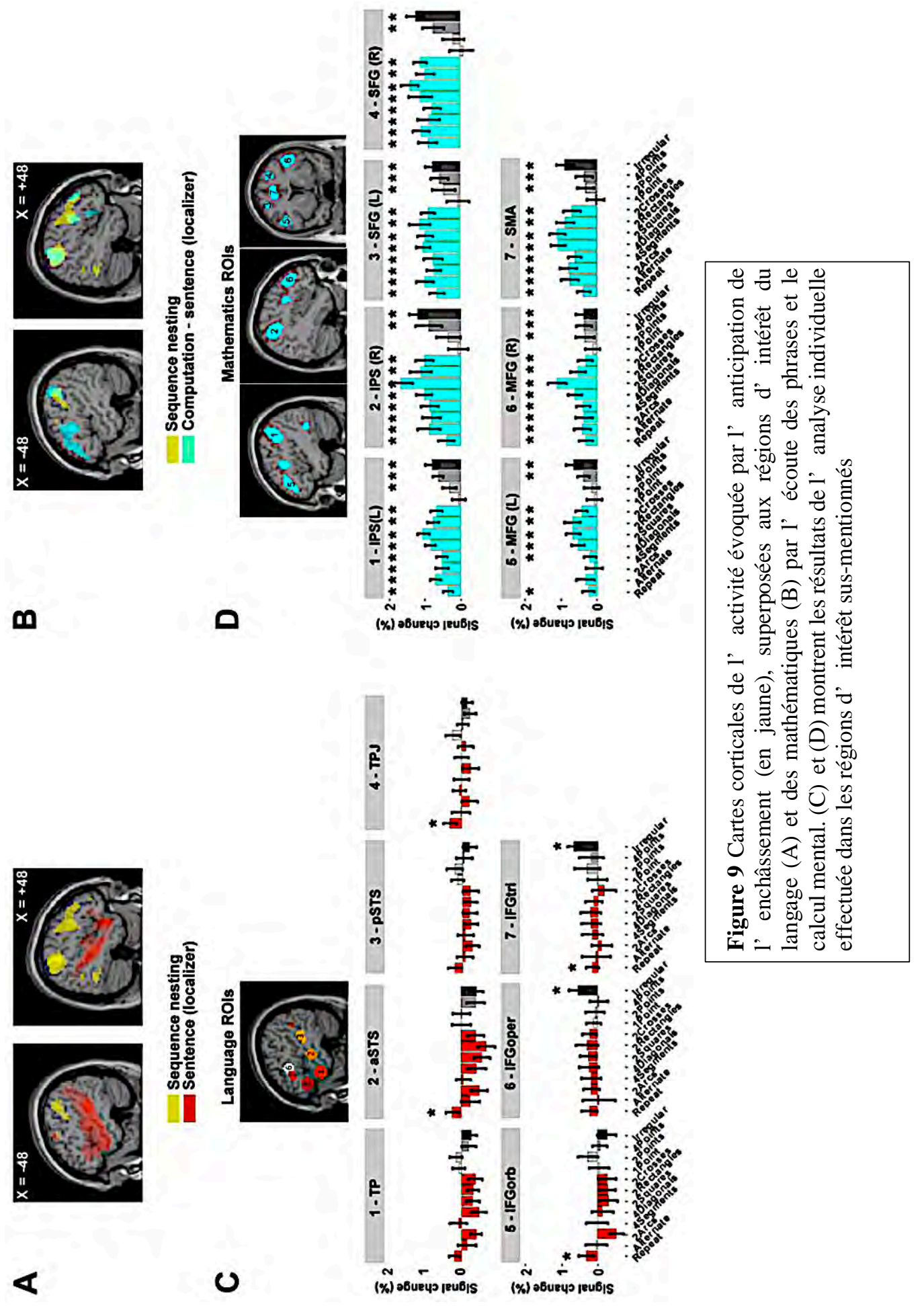


A

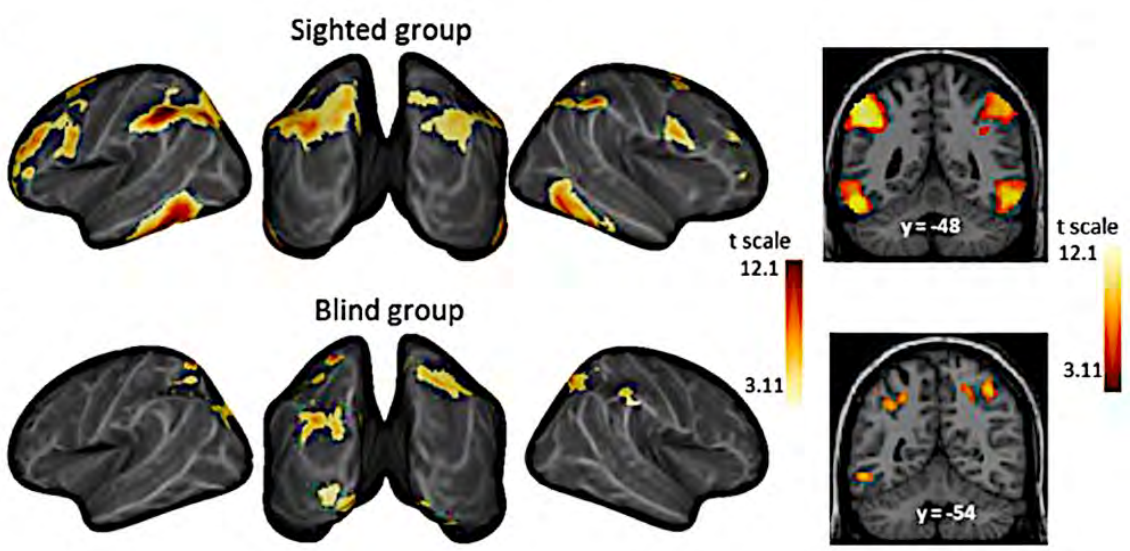

C

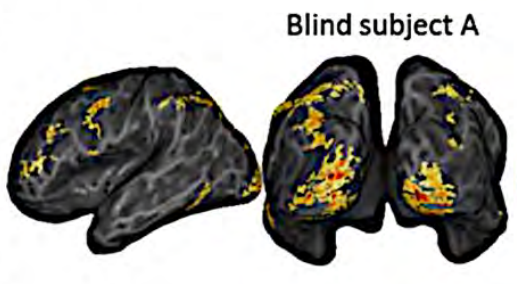

D

\section{Blind subject B}
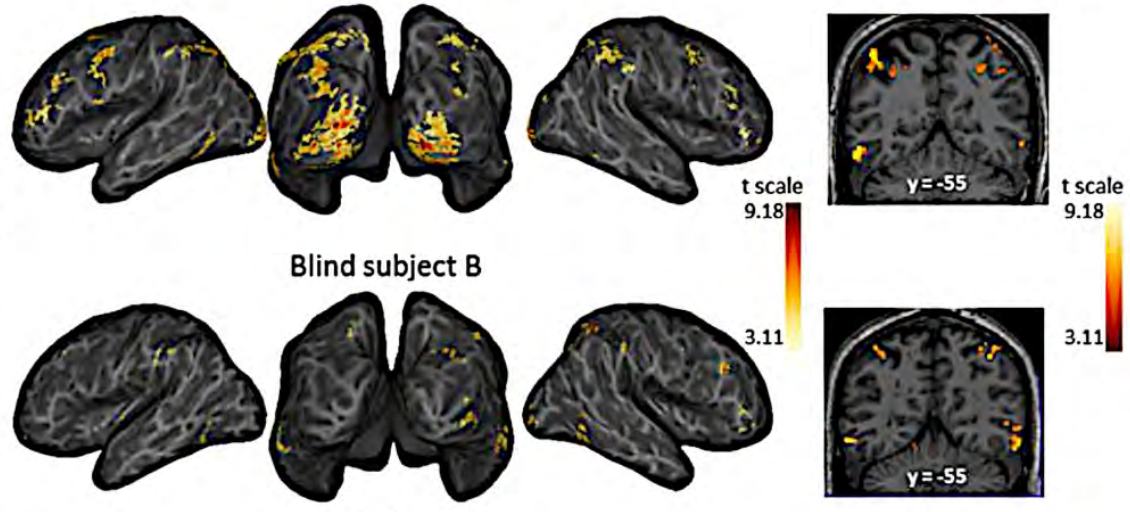

$\mathrm{E}$

Blind subject $C$
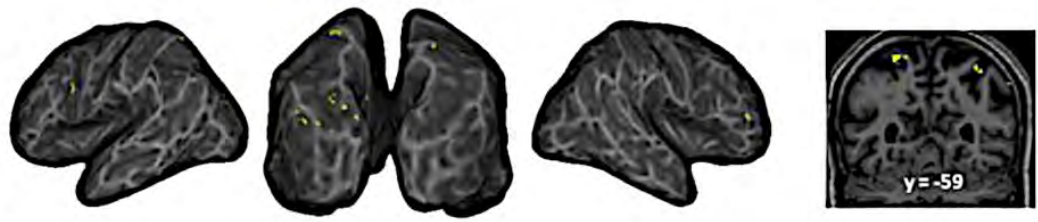

$\mathrm{F}$
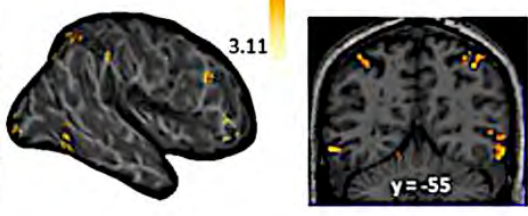

3.11
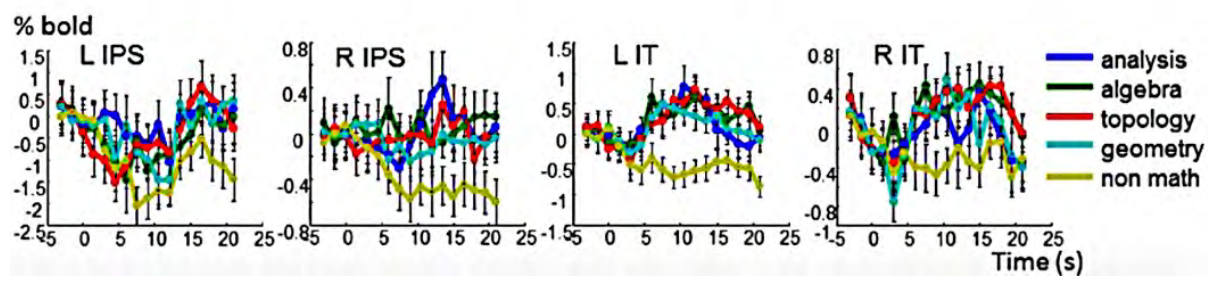

Figure 10 Activations cérébrales pour le contraste math>non-math chez les voyants (première ligne) et les aveugles (quatre dernières lignes). Décours temporel de l'activation chez le sujet aveugle A (troisième ligne) dans les principales régions répondant aux mathématiques, pour différentes disciplines (analyse, algèbre, topologie, géométrie, énoncés non mathématiques).

Page de gauche : Expérience 1. Page de droite : Expérience 2. 
A Sighted group
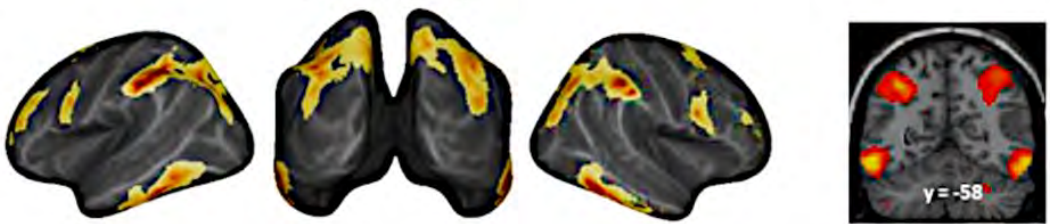

B

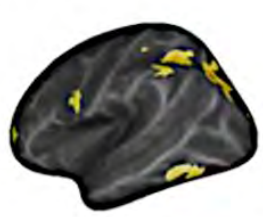

Blind group

C
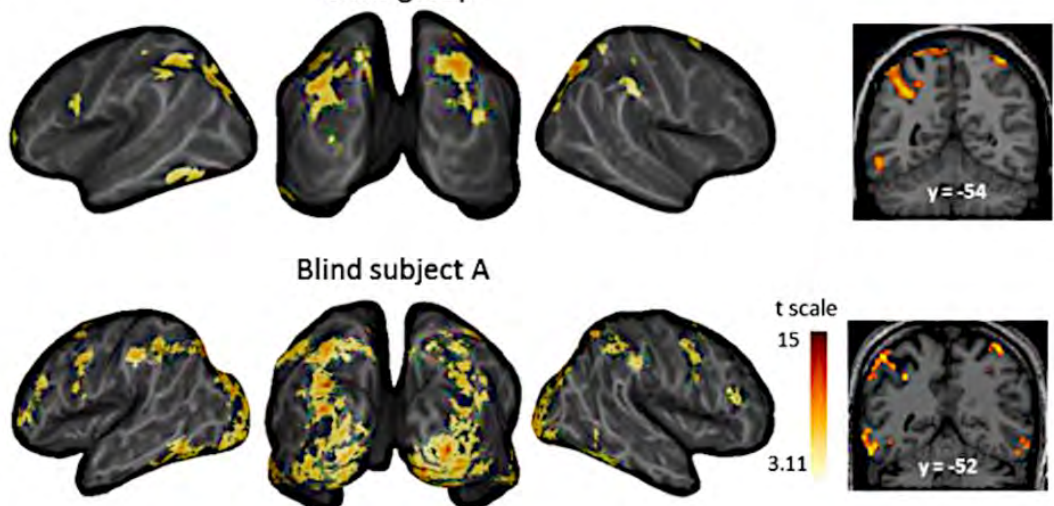

Blind subject A
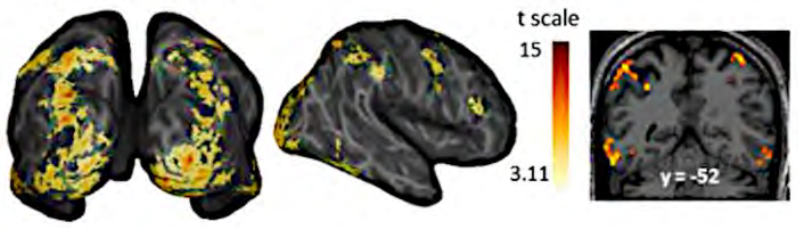

t scale

15

D

Blind subject $B$
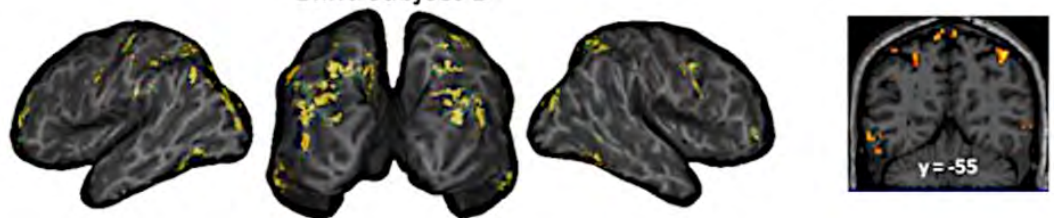

E
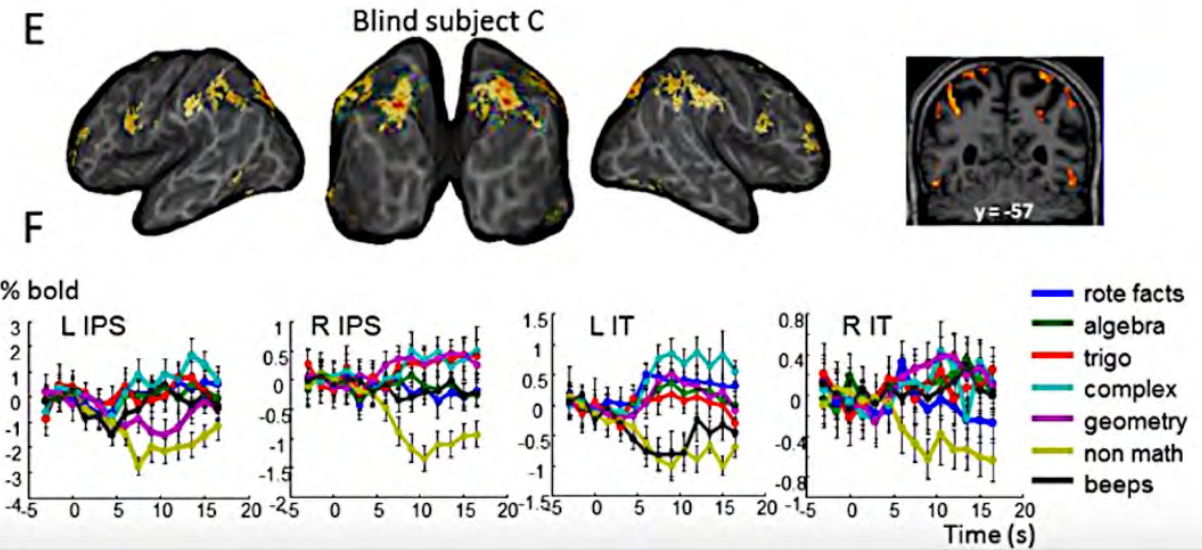


\section{L'expérience visuelle est-elle nécessaire pour développer des connaissances mathématiques (Amalric et al., 2017a)}

Les résultats décrits jusqu'à présent semblent donner raison à l'introspection d'Albert Einstein selon laquelle les mots et le langage n'interviennent pas dans la réflexion mathématique. Avait-il également raison de penser que les images mentales constituent le support privilégié du traitement du contenu mathématique?

Tout d'abord, afin d'évaluer si l'expérience visuelle est requise pour développer des concepts mathématiques avancés, nous avons proposé à trois mathématiciens professionnels non-voyants (les sujets A et B étant devenus aveugles respectivement aux âges de 3 ans et 10 ans, et le sujet $C$ étant aveugle de naissance) de réaliser un examen IRMf utilisant les protocoles des expériences 1 et 2 .

Comme le révèle la figure 10, des activations similaires à celles observées chez les mathématiciens voyants ont été observées chez les mathématiciens non-voyants pour le contraste math > non-math. En particulier dans la seconde expérience, portant sur des concepts plus simples que la première, nous avons pu observer l'activation des sillons intrapariétaux bilatéraux et des régions temporales inférieures bilatérales chez chacun des sujets.

Ces résultats réfutent l'hypothèse d'un lien entre expertise mathématique et expérience visuelle. Au contraire, ils suggèrent que la représentation corticale des mathématiques de haut niveau, qui implique de manière fondamentale les sillons intrapariétaux et les régions temporales inférieures, peut se développer indépendamment de toute expérience visuelle.

Toutefois, nos résultats n'excluent pas la possibilité d'une forme d'imagerie mentale, aussi bien chez les voyants que chez les non-voyants, nécessaire au traitement de certaines notions mathématiques. Tout d'abord, à l'issue de l'expérience 1 , chaque participant a été invité à revoir l'ensemble des affirmations présentées au cours de l'examen IRMf, et à évaluer notamment le niveau d'imagerie visuelle suscitée par chacune d'entre elles. Au sein du groupe des mathématiciens voyants, une région temporale inférieure gauche et une zone du sillon intra-occipital gauche (i.e. deux zones du cortex visuel) ont montré une activation positivement corrélée à la mesure subjective d'imagerie mentale. De plus, dans l'expérience 2, toujours chez les mathématiciens voyants, nous avons observé que des aires cérébrales de traitement visuel telles que la scissure calcarine s'activent lorsque les notions mathématiques abordées impliquent une certaine visualisation de la solution sur le cercle trigonométrique. Enfin, chez les mathématiciens non-voyants, une activation additionnelle par rapport aux voyants a été observée dans le cortex occipital (cf. figure 11). Cette activation est particulièrement intense chez le sujet A qui a également rapporté le plus fort taux d'imagerie mentale à l'issue de l'expérience 1. Même s'il est impossible d'établir une quelconque conclusion sur la base de l'étude d'un groupe de seulement trois individus dont les causes de la cécité sont qui plus est tout à fait différentes, il est tentant de supposer que cette activation occipitale puisse refléter une certaine forme d'imagerie mentale. 


\section{Blind > Sighted}
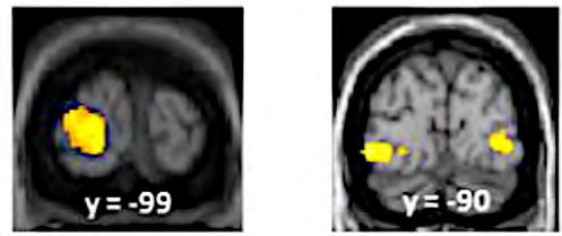

\section{Blind subject $A$}
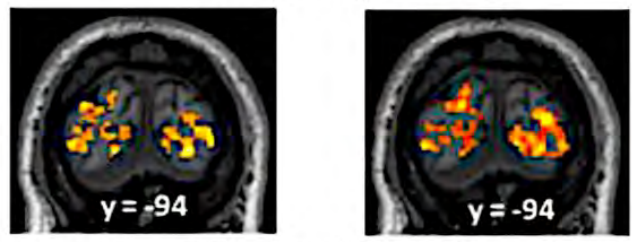

Blind subject $B$

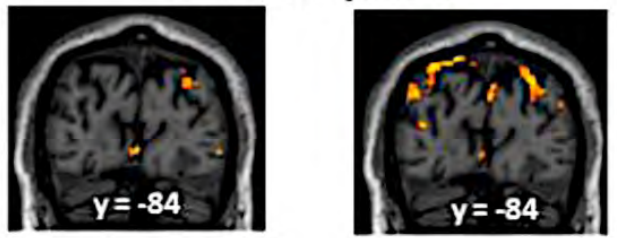

Blind subject $\mathrm{C}$
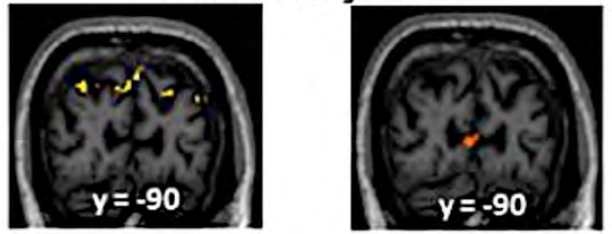

Figure 11 Activation additionnelle du cortex occipital chez les mathématiciens non voyants

\section{Conclusions et perspectives}

Quels supports corticaux pour les mathématiques?

$\mathrm{Au}$ cours de ma thèse, j'ai pu montrer l'existence d'un noyau d'aires cérébrales, bilatéralement composé des sillons intrapariétaux et de régions temporales inférieures, répondant systématiquement aux mathématiques, quels qu'en soient le domaine et le niveau de difficulté, y compris en l'absence d'expérience visuelle. L'ensemble des résultats reportés ici suggère qu'une forme de recyclage neuronal intervient dans le traitement cortical des concepts mathématiques avancés qui semblent se construire sur des fondements proto-mathématiques intuitifs. Les résultats de ma thèse révèlent également que le réseau d'aires répondant aux mathématiques est dissocié des régions cérébrales classiquement impliquées dans le traitement de la syntaxe et de la sémantique du langage. 
Cette dissociation semble intervenir aussi bien au niveau sémantique qu'au niveau syntaxique puisque nous avons pu mettre en évidence l'existence d'une forme de langage de la pensée de nature géométrique, indépendant du langage parlé naturel.

L'idée que cette dissociation puisse refléter l'existence de deux systèmes sémantiques distincts dans le cerveau est même soutenue par les résultats obtenus par Huth et collaborateurs en 2016. En effet, en appliquant de nouvelles méthodes d'analyse de données IRMf enregistrées alors que des participants écoutaient des histoires à contenu très varié, les auteurs ont identifié les deux premières composantes principales de la variation de l'activité cérébrale liée à la sémantique des mots. Or, les deux réseaux sémantiques distincts ainsi identifiés correspondent presque parfaitement aux deux ensembles d'aires cérébrales distincts obtenus en réponse respectivement aux stimuli mathématiques et nonmathématiques dans nos expériences.

\section{Le langage dans l'apprentissage des mathématiques.}

Il est important de noter que les mathématiciens ayant participé à nos études d'imagerie cérébrale avaient tous bénéficié de nombreuses années d'étude préalables des mathématiques. Nous ne pouvons donc que conclure que l'utilisation et le traitement de notions mathématiques bien connues se passent du langage parlé naturel. Toutefois, si on peut supposer qu'une fois acquis, les concepts mathématiques sont encodés de manière abstraite, symbolique, et ne font plus appel au langage, celui-ci pourrait au contraire jouer un rôle important dans leur apprentissage. C'est donc vers l'étude des relations entre mathématiques et langage dans le contexte de l'apprentissage des mathématiques à l'école, que mon travail se tourne désormais.

\section{Quelle est la nature des activités mathématiques?}

Enfin, mes résultats d'IRMf soulèvent de nombreuses questions quant à la définition exacte des processus linguistiques et mathématiques dans le cerveau. Tout d'abord que signifie exactement faire des mathématiques pour le cerveau humain ? Si les sillons intrapariétaux et les régions temporales inférieures s'activent systématiquement en réponse à tout stimulus mathématique, ils ne sont pour autant pas spécifiques des mathématiques et s'activent également dans diverses tâches impliquant un raisonnement logique (Goel and Dolan, 2001; Monti et al., 2009), dans des tests de QI (Duncan, 2010) ou encore dans la représentation de concepts de physique (Mason and Just, 2016). La question reste ouverte de déterminer quel point commun à ces tâches entraîne l'activation de structures cérébrales similaires. Enfin, il est important de remarquer que d'un côté, on ne peut se passer de langage pour communiquer les résultats mathématiques, et de l'autre, le langage courant est envahi de termes mathématiques (nombres, unités, ensembles, quantificateurs, prépositions spatiales, etc...). Où se trouve donc la frontière entre les processus linguistiques et mathématiques dans le cerveau ? 


\section{Références}

Amalric, M., Dehaene, S., 2018. Cortical circuits for mathematical knowledge: evidence for a major subdivision within the brain's semantic networks. Phil Trans R Soc B 373, 20160515. https://doi.org/10.1098/rstb.2016.0515

Amalric, M., Dehaene, S., 2016. Origins of the brain networks for advanced mathematics in expert mathematicians. Proc. Natl. Acad. Sci. 201603205. https://doi.org/10.1073/pnas.1603205113 Amalric, M., Dehaene, S., submitted. A distinct cortical network for mathematical knowledge in the human brain. NeuroImage.

Amalric, M., Denghien, I., Dehaene, S., 2017a. On the role of visual experience in mathematical development: Evidence from blind mathematicians. Dev. Cogn. Neurosci.

https://doi.org/10.1016/j.dcn.2017.09.007

Amalric, M., Wang, L., Pica, P., Figueira, S., Sigman, M., Dehaene, S., 2017b. The language of geometry: Fast comprehension of geometrical primitives and rules in human adults and preschoolers. PLOS Comput. Biol. 13, e1005273. https://doi.org/10.1371/journal.pcbi.1005273 Benson-Amram, S., Heinen, V.K., Dryer, S.L., Holekamp, K.E., 2011. Numerical assessment and individual call discrimination by wild spotted hyaenas, Crocuta crocuta. Anim. Behav. 82, 743752. https://doi.org/10.1016/j.anbehav.2011.07.004

Cantlon, J.F., Merritt, D.J., Brannon, E.M., 2016. Monkeys display classic signatures of human symbolic arithmetic. Anim. Cogn. 19, 405-415. https://doi.org/10.1007/s10071-015-0942-5 Chiandetti, C., Vallortigara, G., 2007. Is there an innate geometric module? Effects of experience with angular geometric cues on spatial re-orientation based on the shape of the environment.

Anim. Cogn. 11, 139-146. https://doi.org/10.1007/s10071-007-0099-y

Chomsky, N., 2006. Language and mind, 3rd ed. ed. Cambridge University Press, Cambridge ;

New York.

Dehaene, S., Cohen, L., 1995. Towards an Anatomical and Functional Model of Number

Processing.

Math. Cogn. 1, 83-120.

Dehaene, S., Izard, V., Pica, P., Spelke, E., 2006. Core Knowledge of Geometry in an Amazonian Indigene Group. Science 311, 381-384. https://doi.org/10.1126/science.1121739 Dehaene, S., Izard, V., Spelke, E., Pica, P., 2008. Log or Linear? Distinct Intuitions of the Number Scale in Western and Amazonian Indigene Cultures. Science 320, 1217-1220.

https://doi.org/10.1126/science. 1156540

Dehaene, S., Piazza, M., Pinel, P., Cohen, L., 2003. THREE PARIETAL CIRCUITS FOR NUMBER PROCESSING.

Cogn. Neuropsychol. 20, 487-506. https://doi.org/10.1080/02643290244000239

Dillon, M.R., Huang, Y., Spelke, E.S., 2013. Core foundations of abstract geometry. Proc. Natl. Acad. Sci.

110, 14191-14195. https://doi.org/10.1073/pnas.1312640110

Duncan, J., 2010. The multiple-demand (MD) system of the primate brain: mental programs for intelligent behaviour. Trends Cogn. Sci. 14, 172-179. https://doi.org/10.1016/j.tics.2010.01.004 Fodor, J.A., 1975. The Language of Thought. Harvard University Press.

Goel, V., Dolan, R.J., 2001. Functional neuroanatomy of three-term relational reasoning.

Neuropsychologia 39, 901-909. https://doi.org/10.1016/S0028-3932(01)00024-0

Hadamard, J., 1975. Essai sur la psychologie de l'invention dans le domaine mathématique. Halberda, J., Mazzocco, M.M.M., Feigenson, L., 2008. Individual differences in non-verbal number acuity correlate with maths achievement. Nature 455, 665-668.

https://doi.org/10.1038/nature07246 
Harvey, B.M., Klein, B.P., Petridou, N., Dumoulin, S.O., 2013. Topographic Representation of Numerosity in the Human Parietal Cortex. Science 341, 1123-1126.

https://doi.org/10.1126/science.1239052

Hermes, D., Rangarajan, V., Foster, B.L., King, J.-R., Kasikci, I., Miller, K.J., Parvizi, J., 2015.

Electrophysiological Responses in the Ventral Temporal Cortex During Reading of Numerals and Calculation. Cereb. Cortex bhv250. https://doi.org/10.1093/cercor/bhv250

Huth, A.G., de Heer, W.A., Griffiths, T.L., Theunissen, F.E., Gallant, J.L., 2016. Natural speech reveals the semantic maps that tile human cerebral cortex. Nature 532, 453-458.

https://doi.org/10.1038/nature17637

Hyde, D.C., Boas, D.A., Blair, C., Carey, S., 2010. Near-infrared spectroscopy shows right

parietal specialization for number in pre-verbal infants. NeuroImage 53, 647-652.

https://doi.org/10.1016/j.neuroimage.2010.06.030

Izard, V., Sann, C., Spelke, E.S., Streri, A., 2009. Newborn infants perceive abstract numbers.

Proc. Natl.

Acad. Sci. 106, 10382-10385. https://doi.org/10.1073/pnas.0812142106

Lee, S.A., Spelke, E.S., 2008. Children's use of geometry for reorientation. Dev. Sci. 11, 743-749. https://doi.org/10.1111/j.1467-7687.2008.00724.X

Marcus, G.F., Vijayan, S., Rao, S.B., Vishton, P.M., 1999. Rule learning by seven-month-old infants.

Science 283, 77-80.

Mason, R.A., Just, M.A., 2016. Neural Representations of Physics Concepts. Psychol. Sci. 27, 904-913. https://doi.org/10.1177/0956797616641941

Matthews, P.G., Lewis, M.R., Hubbard, E.M., 2016. Individual Differences in Nonsymbolic Ratio Processing Predict Symbolic Math Performance. Psychol. Sci. 27, 191-202.

https://doi.org/10.1177/0956797615617799

Monti, M.M., Parsons, L.M., Osherson, D.N., 2012. Thought Beyond Language: Neural

Dissociation of Algebra and Natural Language. Psychol. Sci. 23, 914-922.

https://doi.org/10.1177/0956797612437427

Monti, M.M., Parsons, L.M., Osherson, D.N., 2009. The boundaries of language and thought in deductive inference. Proc. Natl. Acad. Sci. 106, 12554-12559.

https://doi.org/10.1073/pnas.0902422106

Nieder, A., Miller, E.K., 2004. A parieto-frontal network for visual numerical information in the monkey.

Proc. Natl. Acad. Sci. U. S. A. 101, 7457-7462. https://doi.org/10.1073/pnas.0402239101 Pallier, C., Devauchelle, A.-D., Dehaene, S., 2011. Cortical representation of the constituent structure of sentences. Proc. Natl. Acad. Sci. 108, 2522-2527. https://doi.org/10.1073/pnas.1018711108 